\title{
Remote Operations for LHC and CMS
}

\author{
Erik Gottschalk on behalf of the LHC@FNAL Integration Task Force
}

\begin{abstract}
Commissioning the Large Hadron Collider (LHC) and its experiments will be a vital part of the worldwide high energy physics program beginning in 2007. A remote operations center has been built at Fermilab to contribute to commissioning and operations of the LHC and the Compact Muon Solenoid (CMS) experiment, and to develop new capabilities for real-time data analysis and monitoring for LHC, CMS, and grid computing. Remote operations will also be essential to a future International Linear Collider with its multiple, internationally distributed control rooms. In this paper we present an overview of Fermilab's LHC@FNAL remote operations center for LHC and CMS, describe what led up to the development of the center, and describe noteworthy features of the center.
\end{abstract}

Index Terms-Remote operations.

\section{INTRODUCTION}

With the growth of large international collaborations in high-energy physics, it has become essential that collaborators can participate in daily operations of experimental facilities from remote locations. The next big project for high-energy physics is the Large Hadron Collider (LHC), a particle accelerator and collider with a 27 kilometer circumference located at CERN [1]. The LHC provides colliding particle beams for several particle physics experiments, one of which is the Compact Muon Solenoid (CMS) experiment [2] with over 2000 collaborating scientists and engineers. A second experiment, named ATLAS [3], has approximately the same number of scientists and engineers. The large size of these international collaborations is unprecedented, as are the logistics of commissioning and operating the experimental facilities, which are scheduled to begin operating in 2008. For CMS the development of remote operations centers is expected to play an important role in streamlining operations.

Remote monitoring of particle physics experiments is nothing new. This has been done for more than ten years. The next step is to develop remote operations capabilities to enable collaborators to participate in operations from anywhere in the world. The goals are to have secure access to information that is available in control rooms and operations

Manuscript received May 7, 2007. This work was supported in part by Universities Research Association Inc. under Contract No. DE-AC0276CH03000 with the United States Department of Energy.

Dr. Erik Gottschalk is with the Fermi National Accelerator Laboratory, Batavia, IL 60510 USA (phone: 630-840-6416; e-mail: erik@fnal.gov). centers at CERN; safeguards to prevent people working at remote locations from jeopardizing or interfering with operations; collaborative tools for remote participation in shift activities; and eventually remote shifts to streamline operations.

To address the need for remote operations and remote participation in both the LHC accelerator and the CMS experiment, Fermilab has built the LHC@FNAL Remote Operations Center (ROC). Construction was completed in February 2007. The purpose of the ROC is to help scientists and engineers working on LHC and CMS contribute their expertise to commissioning and operations activities at CERN. LHC@FNAL has three primary functions: provide a physical location with access to information that is available in control rooms and operations centers at CERN, serve as a bidirectional communications conduit between CERN and members of the LHC community located in North America, and serve as a focal point for LHC and CMS outreach activities at Fermilab.

\section{BACKGROUND}

Fermilab has been involved in several different aspects of the LHC and CMS. Involvement in both the accelerator and the experiment is perhaps the main reason why the concept of a joint remote operations center shared by both communities evolved at Fermilab.

There are several projects, spanning many years, in which Fermilab has been involved in LHC activities. Fermilab accelerator scientists and engineers have built LHC machine components, Fermilab is involved in several projects tied to the LHC as a collaborating institution in the LHC Accelerator Research Program (LARP) [4], and accelerator scientists, engineers and software professionals from Fermilab have recently become involved in developing software for the LHC. This effort, which is referred to as "LHC@FNAL Software" (LAFS), is directed by the Controls Group [5] of CERN's Accelerators and Beams Department. The Operations Group [6] determines what software is needed and sets the priorities for the LAFS Collaboration.

The LARP and LAFS Collaborations are both interested in remote participation and remote operations for activities at CERN. LARP is interested in remote participation in LHC studies, in providing remote support for LHC accelerator components built in the U.S., and in training people on the LHC controls system before they travel to CERN to work on 


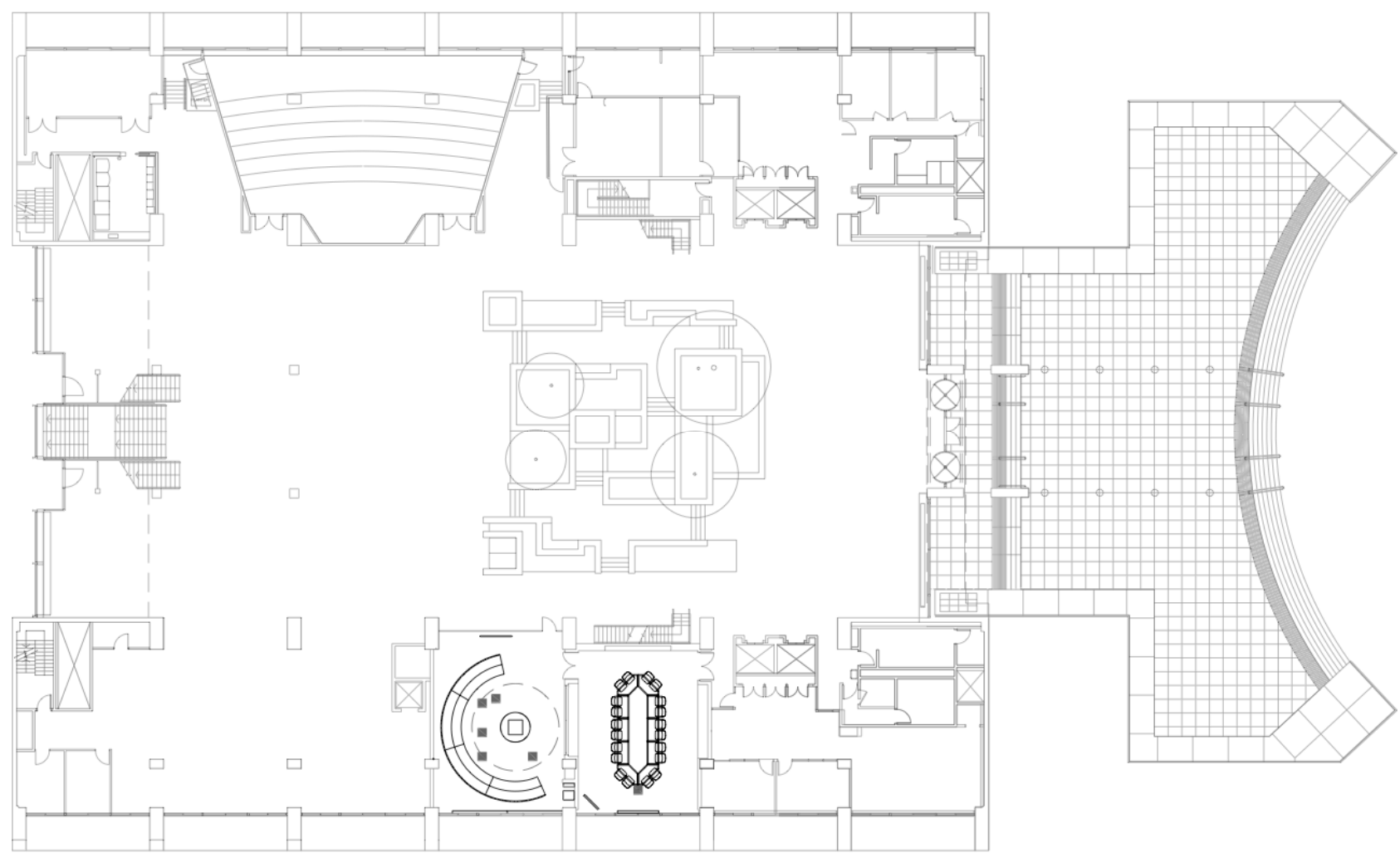

Fig. 1: Floor plan for the $1^{\text {st }}$ floor of Fermilab’s Wilson Hall. The LHC@FNAL remote operations center and conference room are located in the two rooms at the bottom and in the center of the floor plan.

the LHC. The LAFS Collaboration is interested in developing software for the LHC without having to send all of the developers to CERN. Both collaborations have plans to use the ROC.

In addition to involvement with the LHC, Fermilab is the host institution for CMS in the U.S. Scientists and engineers at Fermilab have contributed to CMS detector construction, the lab hosts the LHC Physics Center (LPC) [7] for US-CMS, and it is a Tier- 1 grid computing center for CMS.

With regard to remote operations, the LPC had been planning for many years to establish a remote operations center to be able to provide the space and the tools that are needed for remote monitoring of the quality of the data recorded by CMS. With this plan, a CMS remote operations center was established in 2005 on the $11^{\text {th }}$ floor of Fermilab's Wilson Hall. This remote operations center was used successfully for participation in so-called testbeam activities at CERN, and for the Magnet Test Cosmic Challenges I and II [8]. Construction of the LHC@FNAL ROC began once it was realized that there was an opportunity for accelerator scientists and engineers to work together with CMS experimenters to contribute their combined expertise to the commissioning and operations of LHC and CMS.

\section{LOCATION AND LAYOUT}

The LHC@FNAL ROC and conference room are located at
Fermilab on the first floor of Wilson Hall. Fig. 1 shows a floor plan with the location of the ROC and the conference room at the bottom, center of the figure. The main entrance to Wilson Hall is on the right, a staircase to the main auditorium is on the left, and the Fermilab cafeteria occupies most of the open space to the right of the staircase. By selecting this location for the ROC, the operations center is centrally located and becomes a focal point for LHC and CMS outreach at Fermilab.

The layout of LHC@FNAL is shown in Fig. 2. The ROC is shown on the left. The layout of four consoles is shown, along with screen and projector locations. The wall closest to "CONSOLE 3" was painted with special screen paint and is also used as a projection surface. Four of the projectors are assigned to consoles (one projector per console), and are labeled "PROJ1" through "PROJ4." The fifth projector (labeled "PROJ5") points towards a rear projection screen shown at the top of the figure. This screen, which is referred to as the "Public Display," is used for outreach and has a wide viewing angle so that the display is easily visible from many locations on the first floor. A round table at the center of the room provides additional seating as well as power and network connections for laptop computers.

The LHC@FNAL construction project included installation of a videoconferencing system in the conference room, which is shown on the right side of Fig. 2. A large window was installed between the ROC and the conference 


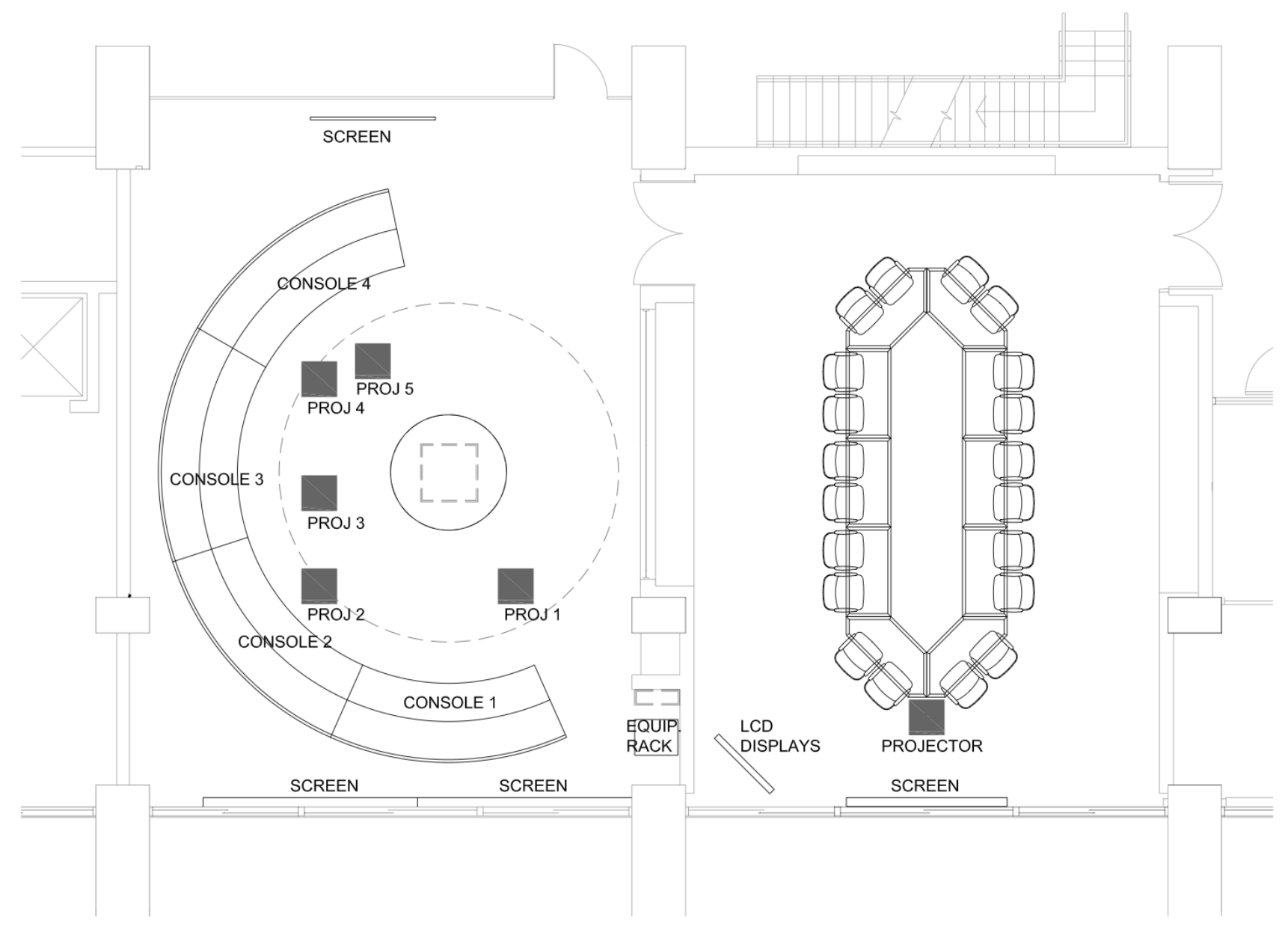

Fig. 2: Layout of the LHC@FNAL remote operations center and conference room. The ROC is shown on the left with the console layout as well as screen and projector locations. The conference room includes two LCD displays, a screen and a projector used for video conferencing.

room so that one would be able to see projector and screen displays in the ROC from the conference room, especially when both rooms are being used for operations. This way the projectors in the ROC can be used to display monitoring information, and participants in the conference room can be engaged in a point-to-point videoconferencing link with participants in a control room at CERN while keeping an eye on the monitoring information. Furthermore, the large window was installed with switchable privacy glass. This provides the option to use the conference room for meetings that require a greater degree of privacy for which a view through the glass is not desired.

The videoconferencing system that was installed is a highdefinition (HD) system. The use of HD videoconferencing in high-energy physics is still relatively new, since reasonably priced systems are just now becoming available. The goal for $\mathrm{HD}$ videoconferencing is to establish a high-quality video and audio link to control rooms and operations centers at CERN and other remote locations. With high-quality video and audio links the ability to be part of activities at CERN is expected to be significantly better compared to standard-definition videoconferencing. The success of this venture into $\mathrm{HD}$ videoconferencing depends on CMS and LHC installing HD equipment at CERN.

Fig. 2 shows the location of the projection screen in the conference room, and the location of a short-throw projector. Two LCD displays in the bottom, left corner of the room are used as video displays for the videoconferencing system. Audio for this system is provided by four ceiling-mounted speakers and microphones (not shown in the figure). All of the electronics for the system are located in an equipment rack that is only accessible from the ROC.

\section{Consoles}

One of the primary functions of LHC@FNAL is to provide a physical location with the means to access and display information as though one were located in an LHC or CMS control room at CERN. One of the ways to achieve this functionality was accomplished by designing the ROC to accommodate consoles that match the specifications of the consoles [9] installed at CERN. This is important, since the 

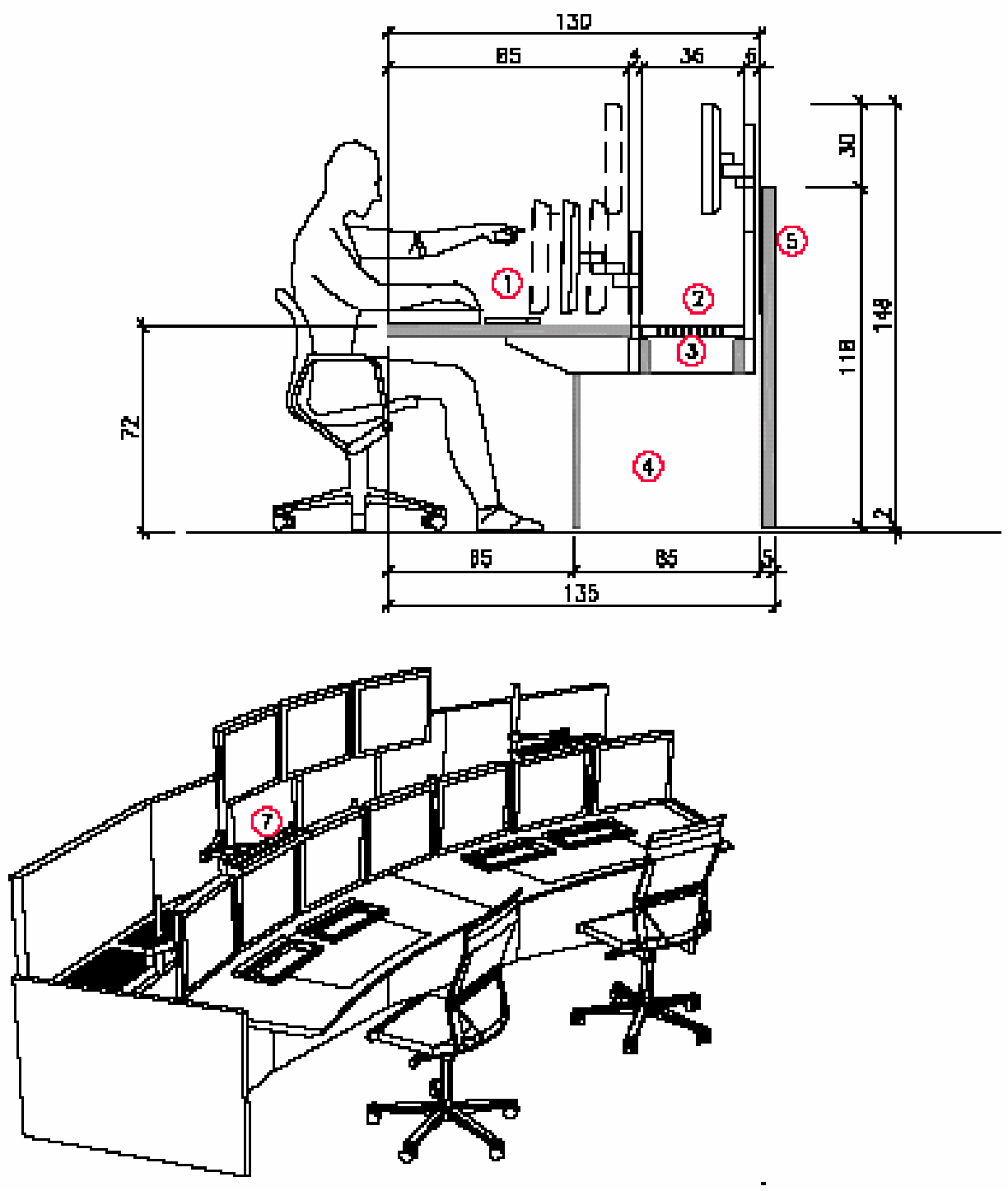

Fig. 3: Two views of an LHC@FNAL console, which was custom-designed for CERN. Dimensions are given in centimeters.

use of consoles with the same specifications makes it possible to implement any changes to the configuration of the consoles that might be made at CERN during the course of LHC and CMS operations.

Each console, as shown in Fig. 3, has two workstations. Each workstation is equipped with a console PC and three LCD screens. The operating system of the PC is configured so that the three screens are treated as a single display with a continuous "desktop." Fig. 3 shows that the tops of the screens are located at eye level on the lower tier of a two-tier mounting system. Located on the upper tier, and centered between the two workstations, are three screens connected to a single so-called fixed-display PC that is used to display monitoring information. Each fixed-display PC also has a projector that has been connected to it. Since the three LCD screens and the projector are treated as a single continuous desktop by the operating system, an operator can easily move a window from the LCD screens to the projector and vice versa. This way a particular window that displays important monitoring information can be brought to the attention of everyone working in the ROC.

In addition to the console PCs and fixed-display PCs, two of the consoles in the ROC are equipped with standalone videoconferencing systems. These are low cost systems that are designed to work well for a few participants who are positioned close to the built-in camera and microphone. We have not had much experience with these systems. The systems seem to be well suited for the ROC since the intended use is for point-to-point videoconferencing between someone in a control room at CERN and someone working at a console 
in the ROC. If these systems turn out to be effective, then we can easily equip each console with its own videoconferencing system.

\section{NETWORK}

The LHC@FNAL network connects to the Fermilab network and therefore to the Internet. It provides access to the CERN network, and to CMS and grid computing resources available at Fermilab. The network consists of two parts with different access restrictions: one with open access that has systems in the ROC connected to the Fermilab network through a gigabit switch, and one with protected access with systems connected through a dedicated router. Systems that are connected to the switch have virtually unrestricted access from off-site and include some of the console PCs and fixeddisplay PCs used by CMS, videoconferencing systems, wireless access points, building environment monitoring system, and the keycard access system. Systems that are connected to the router are protected by a very restrictive access policy implemented with Access Control Lists (ACLs). These systems are on a dedicated subnet that is only available in the ROC. Systems must be registered to use this subnet, thereby preventing people from connecting laptop computers, for example, and gaining access to the protected network.

From the beginning the network for LHC@FNAL was designed with the capability to have a protected network for console PCs, with tightly restricted access into and out of that network. This makes it possible to establish a secure tunnel between the dedicated subnet in the ROC and another network in a remote location, such as a network at CERN. One advantage of establishing a secure tunnel to CERN is that it is more secure than standard methods that are commonly used to connect to systems at CERN.

The LHC@FNAL network is implemented with gigabit uplinks for both the gigabit switch and the router. The switch and the router both support "port channeling," whereby two (or more) Ethernet ports can be combined to share network traffic between the channels. This way the network bandwidth can be easily increased if additional bandwidth is needed for the ROC.

\section{LHC REMOTE OPERATIONS}

As mentioned in Section II of this paper, the LARP and LAFS Collaborations are both interested in using the ROC to work on activities that are based at CERN. For members of the LARP Collaboration, the two primary reasons for participating in the development of LHC@FNAL are to use the ROC for remote monitoring and remote participation. LARP is interested in remote monitoring of key accelerator components that were built by U.S. accelerator scientists and engineers for the LHC, and in remotely participating in LHC commissioning and beam studies.

The LARP planning for remote participation in LHC commissioning is tied to the installation schedule of the LHC. Plans are being developed for participation in injector studies for LHC beam commissioning, currently scheduled for late 2007. A list of requirements for remote participation in such studies is under development. A specific study that is being considered for remote participation is tune feedback in CERN's Super Proton Synchrotron (SPS).

LAFS is interested in using the ROC to work on software development for the LHC. The first project that the LAFS Collaboration started to work on is called Role Based Access Control (RBAC). The software infrastructure for RBAC is crucial for remote operations in that it provides an important safeguard to prevent people from interfering with or even jeopardizing accelerator operations at CERN. For example, with RBAC an accelerator expert who is not in the control room will have the ability to run the accelerator controls system to access a device to monitor its performance. The software infrastructure of the controls system recognizes that the expert is not in the control room, and automatically disables all features of the accelerator controls system that have been deemed to be unsafe for someone outside the control room. In essence, the expert can use the controls system to monitor devices, without having the ability to control the devices. The deployment is scheduled for the end of June 2007.

\section{CMS REMOTE OPERATIONS}

Plans for CMS remote operations involve the CMS control room and multiple remote operations centers. The CMS control room will be located in a surface building at the site of the experiment in Cessy, France. A remote operations center, which is called the "CMS Centre," will be located on the main campus of CERN, in Meyrin. A second remote operations center is the LHC@FNAL ROC at Fermilab. It is likely that additional centers will be built in other locations in the future.

The details of the CMS operations model still need to be worked out, but a very preliminary concept of the division of labor for people in the control room and remote centers has been developed. For the start of CMS operations all control of the CMS detector, trigger and data acquisition systems as well as real-time online monitoring of data quality will be the responsibility of a shift crew working in the CMS control room. Shift crews working in the CMS Centre will be responsible for monitoring data quality and offline computing operations, as well as performing other functions that are not time critical for operations. The remote operations centers, such as LHC@FNAL, are expected to have the same kinds of responsibilities as the CMS Centre. This way some of the shift duties can be transferred from the CMS Centre to remote centers, especially when this entails a reduction in the number 
of people needed for night shifts. The CMS operations model is expected to evolve, and in the future more and more of the responsibilities may be transferred from the control room to the CMS Centre and from there to the remote centers.

For now, the LHC@FNAL ROC is being used by two groups of "early adopters." The first group that started to use the ROC for shift activities is a team of administrators and operators responsible for Tier- 1 grid computing resources at Fermilab. The Tier-1 computing facility at Fermilab operates 24/7 support of critical services and facilities. The primary responder from the administration group resides in the ROC during weekday, business hour shifts, and monitors the functionality of the storage interface, which receives data from CERN and exports data to Tier-2 sites in the U.S. and abroad. The primary responder in the ROC also monitors grid-computing interfaces to processing systems, which accept remote reconstruction, user analysis and simulated event production jobs.

A second group of users is using the ROC for remote shifts for commissioning the CMS silicon tracker. Commissioning of the tracker is done primarily at the Tracker Integration Facility at CERN, and members of the silicon tracker group use the ROC to participate remotely in shift activities by contributing to remote monitoring of the silicon tracker. The efforts of this group are not only contributing to the commissioning efforts at CERN, but are also helping to commission the ROC itself.

There are many monitoring applications that are being developed by CMS collaborators for use in the control room and remote operations centers. In addition to these, the LHC@FNAL Integration Task Force is developing a Screen Snapshot Service (SSS), which is a system to enable remote viewing of displays in a control room or remote operation center. In this system, desktop images of displays of interest are periodically captured and forwarded to a central web server. The server makes the images available to interested remote observers for display in a web browser. SSS is not intended to replace custom remote monitoring applications that are being developed by CMS. Instead it complements them in situations in which there are time or security constraints, or in situations where proprietary applications make a solution difficult to implement.

\section{SUMMARY}

By the end of this decade the majority of high-energy physicists will have turned their attention to the operation of LHC experiments and the LHC accelerator complex. Considering the very large number of people involved in the LHC and its experiments, it has become essential that people be able to participate in daily operations from remote locations. In this context, "remote" is defined to be anywhere outside of a control room. For "remote operations" the goals are to have secure access to information that is available in control rooms; safeguards to prevent people in remote locations from jeopardizing or interfering with operations; collaborative tools to help people work together more effectively; and remote shifts to streamline operations.

To help scientists and engineers working on the LHC and CMS contribute their expertise to commissioning and operations activities at CERN, Fermilab has built the LHC@FNAL Remote Operations Center. Construction of the ROC was completed in February 2007. Two groups of users have started to use the ROC for remote shift activities. The first group is a team of administrators and operators who are responsible for Tier-1 grid computing resources and are using the ROC primarily for monitoring. The second group is participating in remote shifts to commission the CMS silicon tracker. Both groups are providing valuable feedback to help make the ROC more effective for remote operations. Other groups and collaborations working on LHC and CMS have also expressed an interest in using the ROC for their work, and plans for use of the ROC are under development. During the coming months we will develop additional capabilities for remote operations and learn as much as possible from the groups using the ROC to improve our ability to contribute to commissioning and operations of the LHC and CMS.

\section{REFERENCES}

[1] CERN is the European Organization for Nuclear Research (Conseil Européen pour la Recherche Nucléaire). See: http://cern.ch/.

[2] CMS is the Compact Muon Solenoid experiment at CERN. See: http://cms.cern.ch/.

[3] See: http://atlas.web.cern.ch/Atlas/index.html.

[4] LARP is the US LHC Accelerator Research Program, which consists of four US laboratories. See: http://www.agsrhichome.bnl.gov/LARP/.

[5] $\mathrm{AB} / \mathrm{CO}$ is the Controls Group in CERN's Accelerators and Beams Departiment. See: http://ab-div-co.web.cern.ch/ab-div-co/.

[6] $\mathrm{AB} / \mathrm{OP}$ is the Operations Group in CERN's Accelerators and Beams Department. See: http://ab-div.web.cern.ch/ab-div/Groups/OP.html.

[7] LPC is the LHC Physics Center for US-CMS, and it is located at Fermilab. See: http://www.uscms.org/LPC/LPC.htm.

[8] A. Stone, “CMS Remote Monitoring at Fermilab”, unpublished.

[9] Erich Keller AG is the company that designed and built the consoles for CERN. See: http://www.erichkeller.com/de/index.html. 